\section{British Society for Rheumatology}

\section{IMPROVING THE CARE OF YOUNG ADULTS IN RHEUMATOLOGY SERVICES}

${ }^{1}$ Vishit Patel, ${ }^{2}$ Philippa Massouh, ${ }^{2}$ lbrahim Amin, ${ }^{2}$ Emilia Robinson, ${ }^{2}$ Mohammed Thorogood, ${ }^{3}$ Sreena Das. ${ }^{1} N / A$; ${ }^{2}$ King's College London; ${ }^{3}$ King's College Hospital

\subsection{6/archdischild-2021-rcpch.174}

Background Transition clinics facilitate transition to adult services for adolescents and young adults (AYAs). This service for rheumatology patients was audited in 2018/19 to understand their experiences and how it may be improved. Patients reported wanting to see a youth worker and physiotherapist on the same day as transition clinic. They also sought more information about their condition, its management and help in dealing with their emotions.

Objectives To improve the satisfaction of the rheumatology service for AYAs, including providing a good service as described by the AYAs themselves. This quality improvement project (QIP) aimed to achieve this by making changes through three PDSA cycles to the transition clinic these AYAs attend.

Methods The current service was compared to European Alliance of Associations for Rheumatology (EULAR) guidelines on transitional care, which recommend that the service should address psychosocial needs of AYA and provide access to a physical therapist. Additionally, British Society for Paediatric and Adolescent Rheumatology (BSPAR) standards of care for juvenile idiopathic arthritis was also used to consult changes and it was found that AYA should have access to a multidisciplinary team which includes a paediatric clinical psychologist and a physiotherapist. These recommendations were also in the patient's feedback and made them suitable domains for improvement. This led to the introduction of parallel psychology and physiotherapy services to the clinic, achieved by liaising with relevant teams to see if this could be feasible and sustainable. A leaflet was also designed and distributed by the QIP team which included who to contact with health concerns, signposting to information relating to their condition and suggestions of where to go for careers advice or emotional support.

Results 2018-2019 audit data showed that less than 60\% of patients were satisfied with the transition clinic. Following 3 PDSA cycles, satisfaction increased to at least $75 \%$ in the evaluated domains. The addition of a psychologist was well received by patients and the percentage of patients wanting to know where they can get help in dealing with their emotions dropped from $83 \%$ to $75 \%$. Feedback showed patients mostly thought favourably of all evaluated areas of the leaflet. Principally, they found the leaflet satisfactorily explained what transition is, who to contact about health concerns and resources available for emotional support. Evaluation of the parallel physiotherapy service remains to be conducted.

Conclusions The project produced positive results and there was an improvement in patient satisfaction. Some barriers were encountered; the clinics take place once a month, so it was not possible to collect data from the same patients due to the time constraints of the project. A physiotherapist was difficult to arrange due to funding and was not integrated until late into the project, meaning its effect was not evaluated.
Nevertheless, the QIP shows the positive impact of the three interventions and could provide a model for other transition services.

\section{Quality Improvement and Patient Safety}

\section{COOLING FOR TRANSFER: AN INTEGRATED CARE PATHWAY FOR LONDON (COOLTRIP)}

${ }^{1}$ Yinru Lim, ${ }^{2}$ Oliver Walker, ${ }^{2}$ Aishin Lok, ${ }^{2}$ Nandiran Ratnavel. ${ }^{1}$ Neonatal Transfer Service; ${ }^{2}$ Barts Health NHS Trust

10.1136/archdischild-2021-rcpch.175

Background The London Neonatal Transfer Service (NTS) uplifts over a hundred babies each year for cooling treatment for hypoxic ischaemic encephalopathy. We are involved in the stabilisation and transfer of these babies and help support the communication and decision-making process between cooling centres and local units.

In line with the recommendations from the British Association of Perinatal Medicine (BAPM) framework 'Therapeutic hypothermia for neonatal encephalopathy', a timely referral and transfer process with accurate information is necessary.

Objectives We carried out an audit to investigate the quality of documentation of newborn infants transferred between hospitals for cooling, as well as adherence to cooling criteria. We aimed to identify opportunities for service improvement through the audit.

Methods A retrospective audit was carried out. All babies who were transferred within the London network from June 2017 - July 2019 were included. Data collected included time to achieving target temperature, documentation of both Criteria $\mathrm{A}$ and $\mathrm{B}$ outlined in the TOBY study.

Information was obtained from local discharge letters, BadgerNet information and transport documentation.

Results Out of 170 babies, 51 babies (30\%) did not achieve target temperature within the 6 hour recommended window and almost a quarter of these babies (11 of 51 babies) were due to a late referral for transfer.

Focussing on the quality of documentation, Criteria A was well documented. $24 \%$ of babies had incomplete documentation of neurological assessment for Criteria B. Of those who had full documentation of neurological assessment, $45 \%$ did not actually meet Criteria B.

Conclusions The London NTS's unique involvement in the transfer of babies with HIE has allowed us to identify opportunities for service improvement. Our audit revealed that a significant proportion of babies did not have complete documentation of neurological assessment, a key component in the decision making for cooling. There is a lack of uniformity to neurological assessment with no reliable place to transcribe information for transfer between hospitals.

We therefore designed the CoolTrip pathway which is a standardised documentation that helps support local clinical decision making and facilitates accurate record keeping that can be easily transferred between hospitals.

The pathway consists of a decision-making matrix that would enable the local team to identify babies who meets criteria for cooling as set out in the BAPM framework. 
Appropriate and timely discussions with network cooling centre will be triggered with the use of the pathway and documentation of essential information, including a uniform neurological assessment, is ensured. Communication between local teams, cooling centres and with parents will also be clearly documented with the pathway.

This pathway has been accepted and rolled out for use within the London neonatal network and a post-implementation review will be carried out.

\section{British Association of General Paediatrics}

\section{CONSEQUENCES OF DELAYED PRESENTATION OF IRON DEFICIENCY ANAEMIA DURING THE COVID PANDEMIC}

DIAB Osama, Hema Gandecha, Gulmeena Rizvi, Prem Sundaram, Indira Umezurike. Leicester Royal Infirmary Hospital

\subsection{6/archdischild-2021-rcpch.176}

Background Anaemia affects roughly a third of the world's population; half the cases are due to iron deficiency. It is a major and global public health problem that affects child morbidity and mortality. Children aged $0-5$ years are particularly at risk.

Treatment strategies include prevention, through food fortification and iron supplementation, and early detection.

Objectives We present two cases with an insidious presentation and complications of iron deficiency anaemia. We highlight a need to do more to educate parents and carers on the importance of not delaying in seeking medical care, during the current pandemic, as in both cases parents did not feel safe to contact services sooner.

\begin{tabular}{|c|c|c|}
\hline & Case 1 & Case 2 \\
\hline Age & 29 months & 14 months \\
\hline Sex & female & female \\
\hline Ethnicity & Asian & Asian \\
\hline Weight (centile) & $11 \mathrm{~kg}$ (9th-25th percentile) & $9.6 \mathrm{~kg}$ (50th percentile) \\
\hline Symptoms & $\begin{array}{l}\text { Pallor, fatigue and } \\
\text { hypoactivity }\end{array}$ & $\begin{array}{l}\text { Pallor, fatigue and } \\
\text { hypoactivity }\end{array}$ \\
\hline Haemoglobin (gram/l) & 25 & 15 \\
\hline Haematocrit & 0.122 & 0.065 \\
\hline MCV & 49 & 49 \\
\hline Red cell count (*1012/L) & 2.21 & 1.34 \\
\hline White cell count & 11 & 9.7 \\
\hline Platelet count & 843 & 214 \\
\hline Reticulocyte count & 30 & 29 \\
\hline Ferritin (microgram/l) & $<2$ & $<2$ \\
\hline $\begin{array}{l}\text { VitaminB12 (nanogram/ } \\
\text { L) }\end{array}$ & 947 & 912 \\
\hline Folate (microgram/L) & 15.9 & 14.8 \\
\hline Chest X-ray & cardiomegaly & cardiomegaly \\
\hline Echo cardiogram & normal & normal \\
\hline Coeliac antibody & negative & negative \\
\hline Haemoglobin A2 & $1.4 \%$ & $1.3 \%$ \\
\hline Haemoglobin F & $0.9 \%$ & $1.1 \%$ \\
\hline
\end{tabular}

Methods Two cases aged 14- and 29-months-old Asian (Indian) girls who presented to the Emergency Department, with sever pallor, tiredness and lack of activity/energy. History revealed that both had been pale for several months; however, parents were hesitant to seek medical advice because of the fear of exposure to corona virus infection.

Full blood count (FBC) showed haemoglobin levels of 16 and $29 \mathrm{~g} / \mathrm{l}$ respectively.

Other causes like haemoglobinopathy, malignancy and infection were excluded, and the diagnosis of Iron deficiency anaemia was confirmed.

They both had received blood transfusion which was easily avoidable. Fortunately, they were not showing any signs of high output cardiac failure and after four days they were discharged home safely.

Results

Conclusions Delayed presentation of easily preventable health issues is clearly still a problem, during the current pandemic, despite efforts made by health organisations. The message needs to be reiterated and further steps need to be taken towards health education and public orientation through different multimedia messaging to encourage people to seek medical advice early when needed. due to the long-lasting nature of this pandemic, minor problems that may not have been concerning a year ago may now manifest, if left untreated, as serious, or critical illness. It is our collective role as paediatricians and health care professionals to help prevent this occurring.

\section{British Association of Perinatal Medicine and Neonatal Society}

\section{REVIEW OF PRACTICES AROUND PERINATAL SENTINEL EVENTS OF PRETERM BABIES WITH SEVERE NEUROLOGICAL INJURY IN A SINGLE TERTIARY CARE CENTRE IN CANADA}

Sriya Roychaudhuri, Joseph Ting, Mimi Kuan, Natalie Chan. BC Women and Children's Hospital, Vancouver, Canada

\subsection{6/archdischild-2021-rcpch.177}

Background Preterm infants born $<33$ weeks gestational age (GA) are at risk of intraventricular hemorrhage (IVH) and periventricular leukomalacia (PVL) in the early peri-natal period. Grades 3 and $4 \mathrm{IVH}$ and PVL are considered severe neurological injury (SNI) and the prevalence of neurodevelopmental disability in survivors is high.

Objectives This project aims to analyze practices and trends over a 10 years span, around major sentinel events, which are known to have contributory role in causality of SNI in preterm babies, in a single tertiary care, level 3 NICU with a higher rate of SNI compared to the national annual rates according to the Canadian Neonatal Network (CNN).

Methods The nation-wide Evidence Based Practice for Improving Quality (EPIQ) Guidelines have an enlisted set of sentinel events with levels of recommendations spanning multiple disciplines, to reduce the SNI in this population. We assessed practices around each antenatal, intrapartum and early postnatal sentinel event. A total of 42 sentinel 